\title{
Morphological characteristics of clinically significant coronary artery stenosis in stable angina
}

\author{
J R W HANGARTNER, A J CHARLESTON,* M J DAVIES, A C THOMAS \\ From the British Heart Foundation, Cardiovascular Pathology Unit, St George's Hospital Medical School, \\ London
}

SUMMARY All segments of clinically significant stenosis in the coronary arteries of 54 men with stable angina were categorised according to the position of the plaques (eccentric or concentric) and the presence or absence of a pool of extracellular lipid. In the group as a whole, stenosis of $>50 \%$ by diameter was caused by concentric fibrous plaques in $48 \%$ of lesions, by concentric lipid plaques in $28 \%$, by eccentric fibrous plaques in $12 \%$, and by eccentric lipid plaques in $12 \%$. In addition, 43 of the 54 patients had one or more stenoses with multiple channels (recanalisation). Eccentric plaques with an arc of normal vessel wall occupying more than $16 \%$ of the circumference of the residual lumen were considered to have a vasospastic potential and made up $15 \%$ of all lesions with stenosis of $>50 \%$ by diameter. Forty four per cent of plaques causing stenosis between $30 \%$ and $50 \%$ by diameter were eccentric and retained a considerable arc of normal media. These lesions were often in series with segments of higher grade stenosis that did not have an arc of normal media. The overall frequency of plaque types gave no indication of the proportions of different plaque types within an individual. In $15 \%$ of patients all the plaques causing $>50 \%$ diameter stenosis were fibrous and in $13 \%$ of patients all the plaques were of the lipid type. Most patients had mixtures of all plaque types in varying proportions. Plaques with a large pool of lipid were not found in $33 \%$ of patients whereas they formed $>90 \%$ of the plaques in $9 \%$ of patients. No segments of stenosis $>50 \%$ by diameter with a vasospastic potential were found in $44 \%$ of the patients but one or more such plaques was present in the remaining $56 \%$. Three patients $(6 \%)$ each had five separate segments of stenosis with a vasospastic potential.

The results indicate that even in a population of men with stable angina in whom diabetes is excluded the distribution of types of atheromatous lesions is very heterogenous.

In vivo coronary angiograms demonstrate that pharmacological intervention can lead to significant changes in the diameter of the residual lumen in some stenotic coronary arteries. ${ }^{1}$ The variation of lumen diameter showed by such coronary angiograms and referred to as "dynamic stenosis" has been reviewed ${ }^{2}$ and is thought to occur in the presence of plaques that are eccentric and thus retain an arc of arterial wall containing medial muscle capable of contraction. While stenotic segments with such a

Requests for reprints to Dr J R W Hangartner, Department of Histopathology, St George's Hospital Medical School, Cranmer Terrace, London SW17 0RE.

^Present address: University of Auckland Medical School, Auckland, New Zealand.

Accepted for publication 29 July 1986 vasospastic potential have been reported to make up $70 \%$ of all high grade stenosis ${ }^{3-5}$ in other pathological studies they are reported to be rare. ${ }^{6}$ This form of stenosis has been confirmed to be the cause of some cases of unstable angina ${ }^{7}$ and is a possible explanation for spontaneous episodes of silent myocardial ischaemia unrelated to exercise in patients with stable angina. ${ }^{8}$ Case selection may be responsible for the variation in the reported incidence of stenoses with a vasospastic potential and further data are needed to determine whether such lesions are present in all patients with advanced coronary atheroma or are confined to a minority.

The configuration of stenotic segments of coronary artery is also relevant to angioplasty. Clinical studies suggest that some lesions can be dilated by much lower pressures than others and this may imply that there is more than one type of stenosis. ${ }^{49}$ 
For these reasons the present study is concerned with defining the morphological appearances, at necropsy, of segments of high grade stenosis, as seen in transverse sections of arteries perfused at systemic pressure, in patients who had a clinical diagnosis of stable angina in life.

\section{Patients and methods}

\section{PATIENTS}

The study group consisted of 54 men aged 45-69 (mean 59.9) in whom the clinical diagnosis was stable angina. All died suddenly outside hospital within six hours of the onset of symptoms in their last attack, none had consulted a doctor within the previous two weeks, and all had been able to lead a normal active life. A detailed postmortem examination including coronary angiography showed no other cause of death other than stenosis caused by atheroma of $>75 \%$ by cross sectional area $(50 \%$ diameter) in one or more segments of coronary artery. A small number of cases with stable angina found to have aortic valve stenosis at necropsy were excluded from the study. Women were excluded because there were too few of them; patients with known diabetes were also excluded. Evidence of previous healed myocardial infarction, in the form of macroscopic regional scarring, was present at necropsy in 38 of the 54 cases. All the patients has been receiving medical treatment for the control of exercise induced angina for 1-13 years (mean (SD) 4.8 (3.6) years). The patients had not had ambulatory monitoring to detect silent ischaemic attacks but any patient who had been diagnosed as having unstable angina in the last six months was excluded. Four patients had had selective coronary arteriography in life.

\section{METHODS}

Postmortem coronary arteriography was performed in all cases. The technique and subsequent processing and histological examination of the major epicardial coronary arteries have been described. ${ }^{1011}$

In brief, the method enables detailed histological examination and quantification of representative sections of $3 \mathrm{~mm}$ segments of the entire epicardial coronary artery tree, which has been distended at physiological pressure by the controlled injections of a mixture of gelatin and barium sulphate before fixation. Subsequently the position of each segment within the coronary artery tree was determined from postmortem arteriograms. Segments were coded by the modified Greenlane Hospital system. ${ }^{12}$ Sections of each $3 \mathrm{~mm}$ segment of coronary artery were stained with an elastica/haematoxylin and eosin stain.

\section{MORPHOLOGICAL DEFINITIONS}

Examination of histological cross sections of coronary arteries that have been distended before fixation allows a simple morphological classification of stenotic segments. Any segment of stenosis may be due to eccentric (figs 1 and 2) or concentric (figs 3 and 4) intimal thickening; the former may be associated with a vasospastic potential. Any segment of stenosis may consist predominantly of intimal fibrosis (figs 2 and 3 ) or contain, in addition, a pool of free extracellular lipid containing cholesterol and its esters (figs 1 and 4). It is the latter type that has been described as "soft", with the lipid pool being separated from the vessel lumen by a cap of fibrous tissue. The fibrous plaque may contain small amounts of intracellular lipid but the special feature of the lipid rich plaque is a space within the intima containing extracellular lipid. Segments of stenosis in which the original lumen is filled by fibrous tissue containing several new vascular channels (fig 5) are regarded as representing restoration of flow by organisation rather than by lysis of the previous occlusive thrombus.

A plaque was defined as eccentric if there was an associated arc of normal artery wall (figs 1 and 2). The intima was regarded as normal when it was less thick than the media. The thickness of the media and intima were measured perpendicularly to the internal elastic lamina. When the arc of normal arterial wall was $\geqslant 16 \%$ of the perimeter of the residual lumen (fig 6) a potential for vasospasm was presumed. We chose $16 \%\left(60^{\circ}\right.$ of an arc of vessel perimeter) to make the data comparable with previous studies. ${ }^{3}$ Segments of stenosis in which there was no

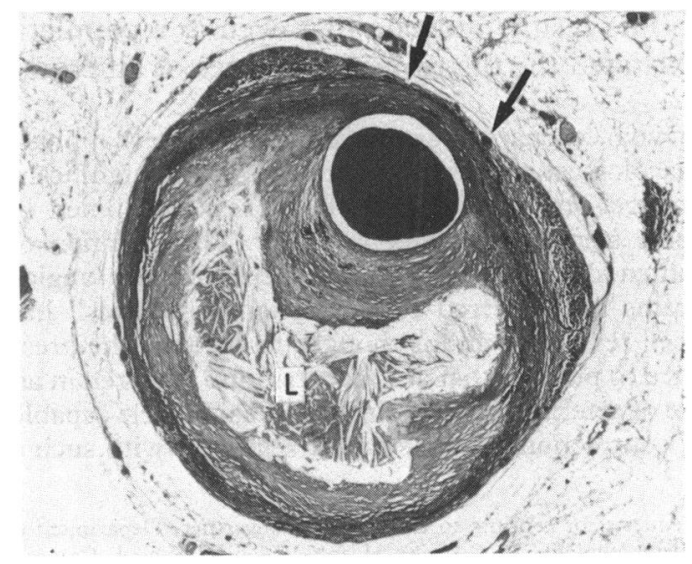

Fig 1 Photomicrograph of transverse section of stenosis caused by an eccentric lipid rich plaque. The lumen contains angiographic medium. An arc of normal vessel wall is indicated (arrows). The thickened intima contains a pool of extracellular lipid (L). Elastica/haematoxylin and eosin stain. 
arc of normal arterial wall were defined as concentric (figs 3 and 4). Plaques with a pool of extracellular lipid within the intima were defined as lipid in type (figs 1 and 4). If an extracellular pool of lipid was not present the plaque was defined as fibrous. Segments in which there were several vascular channels within the original lumen (fig 5 ) were recorded but the degree of stenosis was not measured.

\section{MEASUREMENT}

Stained sections of the coronary arteries were measured with a microscope linked to a digitising bit pad and computer. All the sections from each case were measured by two observers. The perimeters of the

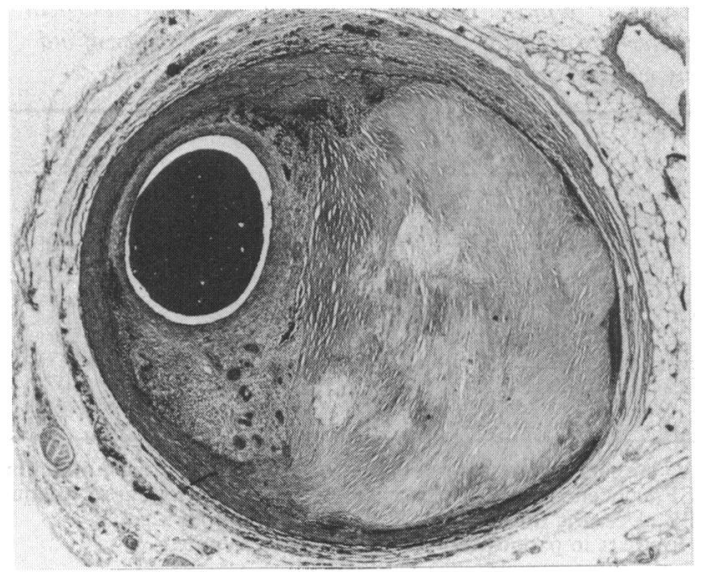

Fig 2 Photomicrograph of transverse section of stenosis caused by an eccentric fibrous plaque. There is an arc of normal media adjacent to the lumen. The plaque does not contain a pool of lipid. Elastica/haematoxylin and eosin stain.

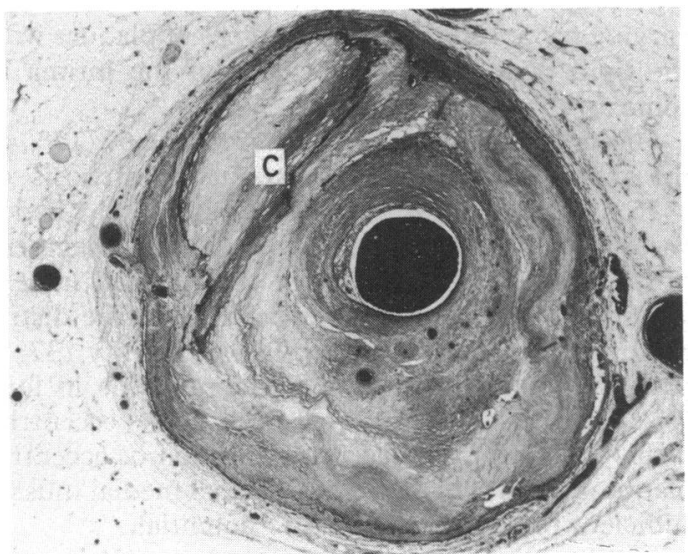

Fig 3 Photomicrograph showing stenosis caused by concentric fibrous thickening of the intima. The small central residual lumen contains angiographic medium. The intima does not contain a lipid pool but there is a plate of calcification (C). Elastica/haematoxylin and eosin stain. internal elastic lamina and residual lumen were measured. All stable plaques (that is those without thrombosis in which the stenosis calculated from the perimeter measurements was $\geqslant 50 \%$ of the cross sectional area corresponding to $30 \%$ stenosis or greater by diameter) were coded for the morphological characteristics as defined above. When they were present the circumferential length of arcs of residual normal arterial wall and the area of the lipid pool were also measured.

\section{STATISTICAL METHODS}

The proportion of the different morphological types of plaques were compared by the $\chi^{2}$ test, corrected

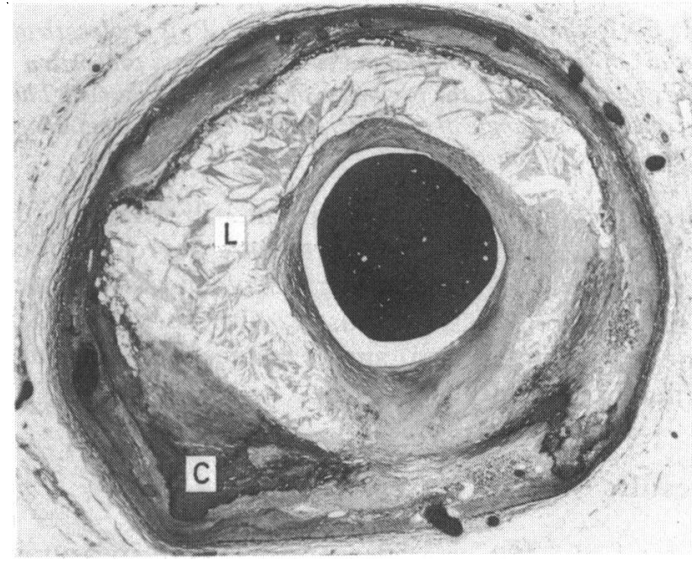

Fig 4 Photomicrograph showing stenosis caused by concentric lipid rich plaque. A pool of lipid $(L)$ almost surrounds the residual lumen. Calcification $(C)$ is also present. Elastica/haematoxylin and eosin stain.

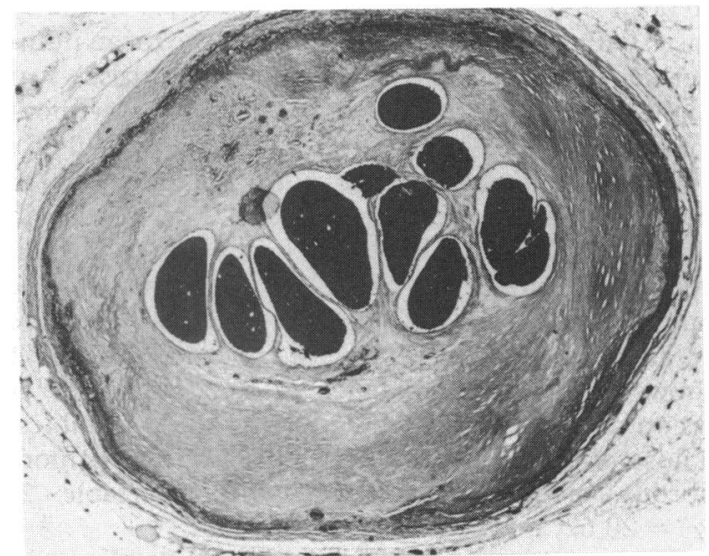

Fig 5 Photomicrograph showing recanalisation of a stenosis. The intima is replaced by connective tissue within which there are nine new vascular channels each containing angiographic medium. Elastica/haematoxylin and eosin stain. 


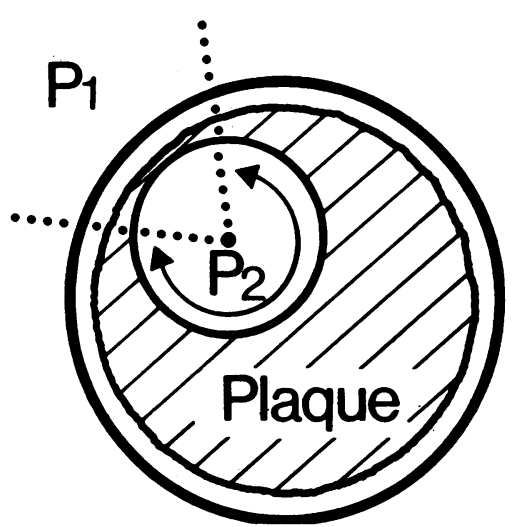

Fig 6 Definition of vasospastic potential. $P_{1}$ is the length of the arc of the normal media and is expressed as a proportion of $P_{1}$ plus $P_{2}$, that is the perimeter of the residual lumen. The arc subtends an angle of $60^{\circ}$ if $P_{1}$ is $16 \%$ of the circumference of the residual lumen.

for continuity where appropriate. Probability (p) values are given for the appropriate degree of freedom. Means (1 SD) are also given when appropriate. The unpaired $t$ test was used, with correction for unequal sample size and variance, to compare differences in means.

\section{Results}

A total of 3480 arterial segments were measured from the 54 hearts.

\section{STENOSES $>75 \%$ BY CROSS SECTIONAL AREA ( $50 \%$ BY DIAMETER)}

Four hundred and forty eight arterial segments had stenosis of $>75 \%$ by cross sectional area. In the 38 cases with healed regional infarction the mean number of stenotic segments (9.5(6.9) per case) was higher than the mean number in cases without infarction (5.6 (3.3) per case) $\left(t^{\prime}=2.804, p<0.05\right)$.

When all 54 cases were considered $270(60.3 \%)$ of the $\mathbf{4 4 8}$ plaques were fibrous and 178 were lipid $(39.7 \%)$ with a large lipid pool occupying more than $10 \%$ of the intima in most $(27.9 \%$ of the total). One hundred and six $(24 \%)$ of the 448 plaques were eccentric and $342(76 \%)$ were concentric. Table 1 shows the overall incidence of the four types of lesion representing combinations of these variables. Those with old infarction had significantly more fibrous plaques than those without (table 2) $\left(\chi^{2}=20.62, \mathrm{p}<0.005\right)$.

There was considerable variability in the distribution of the different morphological types of stenosis between individuals. Eight $(15 \%)$ of patients had only fibrous plaques while seven (13\%) patients had
Table 1 Overall distribution of plaque types in 54 men with stable angina

\begin{tabular}{lll}
\hline & $\%$ stenosis by cross sectional area \\
\cline { 2 - 3 } & $\geqslant 50$ to $<75 \%$ & $>75 \%$ \\
\hline CF & $\left.\begin{array}{l}282(41) \\
\text { CL }\end{array}\right\} 385(56)^{\star}$ & $\left.\begin{array}{r}216(48) \\
126(28)\end{array}\right\} 342(76)^{\star}$ \\
EF & $\left.\begin{array}{r}103(15) \\
\text { EL }\end{array} \quad \begin{array}{l}54(12) \\
52(12)\end{array}\right\} 106(24)^{\star}$ \\
\hline
\end{tabular}

$\star \chi^{2}=50.02 ; \mathrm{p}<0.005$.

CF, concentric fibrous; CL, concentric lipid; EF, eccentric fibrous; EL, eccentric lipid.

Numbers in parentheses are percentages (rounded).

Table 2 Number of segments showing each morphological pattern by grade of stenoses and presence or absence of old myocardial infarction

\begin{tabular}{|c|c|c|c|c|}
\hline & $\%$ stenos & cross secti & area & \\
\hline & $\geqslant 50$ to $<$ & & $>75 \%$ & \\
\hline & $\bar{A}$ & $A I$ & $\bar{A}$ & $A I$ \\
\hline $\begin{array}{l}\text { CF } \\
\text { CL } \\
\text { EF } \\
\text { EL }\end{array}$ & $\begin{array}{l}67(38) \\
26(15) \\
42(24) \\
43(24) \\
\chi^{2}=8.1\end{array}$ & $\begin{array}{r}215(44) \\
77(15) \\
146(28) \\
77(15) \\
<0.05\end{array}$ & $\begin{array}{l}26(29) \\
39(43) \\
10(11) \\
15(17) \\
\chi^{2}=20 .\end{array}$ & $\begin{array}{c}190(53) \\
87(24) \\
44(12) \\
37(10) \\
\mathrm{p}<0.005\end{array}$ \\
\hline
\end{tabular}

A, Angina alone; AI, angina and old myocardial infarction; $\mathrm{CF}$, concentric fibrous plaque; $\mathrm{CL}$, concentric lipid plaque; $E F$, eccentric fibrous plaque; EL, eccentric lipid plaque.

Numbers in parentheses are percentages (rounded).

virtually all lipid plaques and very few fibrous plaques (fig 7). In five ( $9 \%$ ) patients plaques with a large pool of lipid (fig 8) were the only lesions whereas $18(33 \%)$ patients had no lesions of this type. Between these extremes were patients with varying proportions of the two types of plaques with the lipid pool occupying $>10 \%$ of the intima in some.

Of the 106 plaques that were eccentric $69(15 \%$ of the total number of plaques causing stenosis of $>75 \%$ of the cross sectional luminal area) had a vasospastic potential as defined as a medial segment of $\geqslant 16 \%$ of the perimeter of the residual lumen. There was also considerable variation in the distribution of eccentric plaques (fig 9). Twenty (37\%) patients had no plaques of this type while in four (7\%) patients all of the plaques were eccentric. Table 3 shows the uneven distribution of eccentric plaques with a retained arc of normal medial muscle sufficient to allow a vasospastic potential.

STENOSES $>50 \%$ BUT $<75 \%$ BY CROSS

SECTIONAL AREA

Cases with angina and old myocardial infarction had more stenotic segments of this degree $(13.6(7 \cdot 1)$ per 
case) than those with angina alone $(11 \cdot 1(4 \cdot 8)$ per case), although this difference was not significant $\left(t^{\prime}=1.504, p<0.05\right)$. Of the total of 693 plaques with this degree of stenosis, $470(67.8 \%)$ were fibrous and 223 were lipid $(32 \cdot 2 \%)$. Eccentric

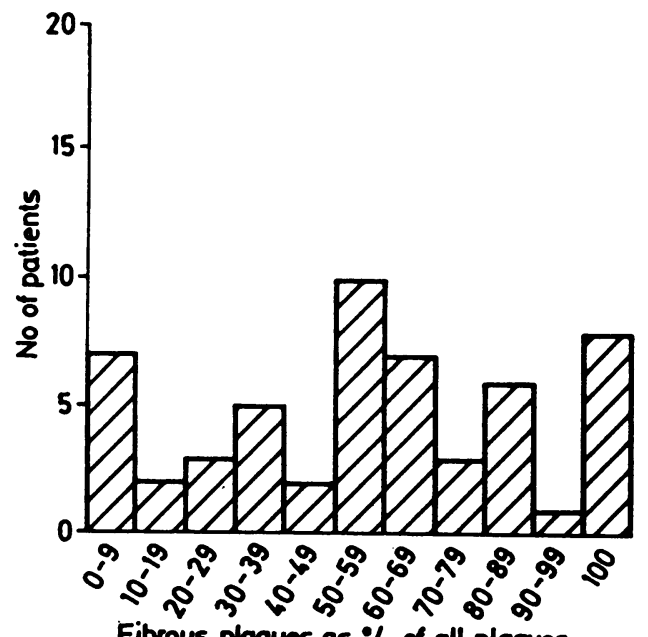

Fig 7 Histogram showing the number of patients in the whole study $(n=54)$ with fibrous plaques that caused $>75 \%$ stenosis by cross sectional area. The results are expressed as a percentage of the total number of plaques per case causing $>75 \%$ stenosis by cross sectional area. Interval ranges are indicated.

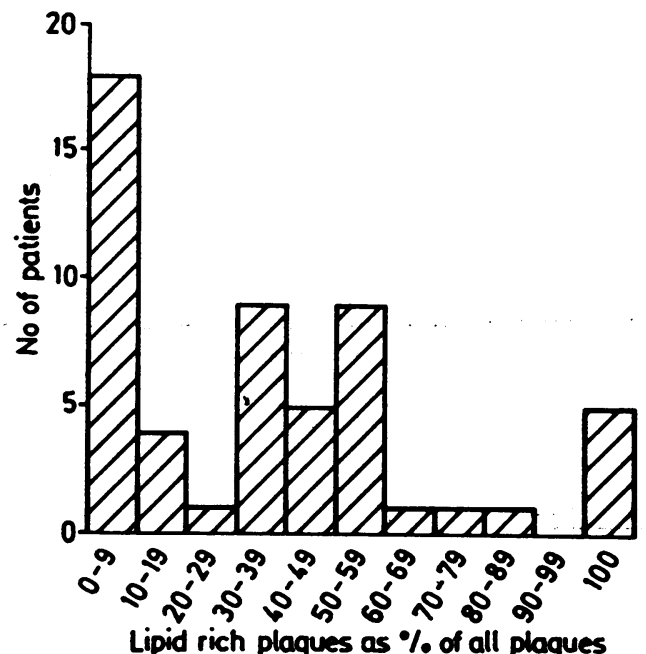

Fig 8 Histogram showing the number of patients in the whole study $(n=54)$ with lipid rich plaques that caused $>75 \%$ stenosis by cross sectional area. The results are expressed as a percentage of the total number of plaques per case causing $>75 \%$ stenosis by cross sectional area. A lipid rich plaque is defined as a lipid pool occupying $>10 \%$ of the intima area. Interval ranges are indicated. plaques made up $308(44 \%)$ of the 693 plaques. As for the higher grade lesions there was a difference in the distribution of the morphological types of plaque (table 2) $\left(\chi^{2}=8.16, p<0.05\right)$ between those with and those without old myocardial infarction.

\section{COMPARISON OF HIGH AND LOW GRADE STENOSIS}

The proportion of eccentric plaques becomes lower, and conversely the proportion of concentric plaques higher, with increasing stenosis $\left(\chi^{2}=50.02\right.$, $\mathrm{p}<0.005)$. The proportional rise in concentric lipid plaques is greater than that of concentric fibrous plaques. Patients with evidence of old myocardial infarction showed significantly more concentric fibrous plaques, particularly at high grade stenosis, than patients without evidence of old myocardial infarction (table 2).

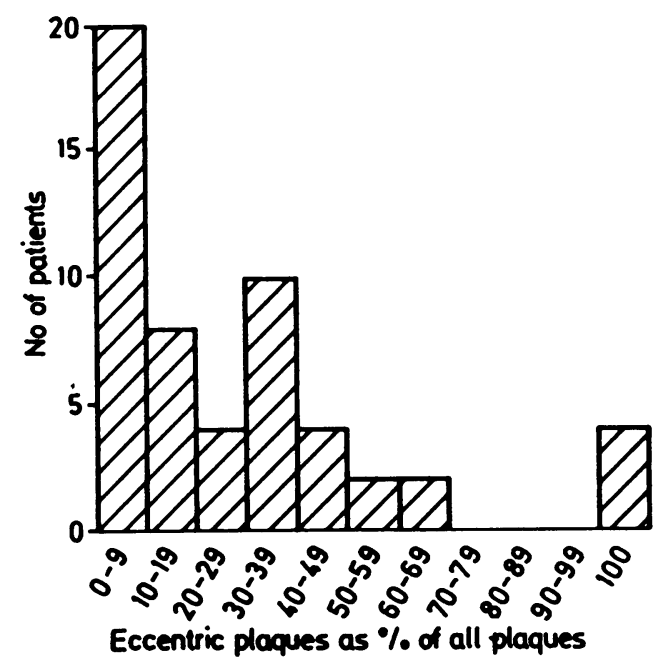

Fig 9 Histogram showing the number of patients in the whole study $(n=54)$ with eccentric plaques (both fibrous and lipid) causing $>75 \%$ stenosis by cross sectional area. Results are expressed as a percentage of the total number of plaques per case causing stenosis $>75 \%$ by cross sectional area. Interval ranges are indicated.

Table 3 Number of cases with segments having a vasospastic potential and $>75 \%$ stenosis by cross sectional area

\begin{tabular}{lllllll}
\hline \multicolumn{6}{c}{ Number of segments } \\
\cline { 2 - 7 } & 0 & 1 & 2 & 3 & 4 & 5 \\
\hline $\begin{array}{l}\text { No of cases } \\
(\mathrm{n}=54)\end{array}$ & $24(44)$ & $12(22)$ & $7(13)$ & $4(7)$ & $4(7)$ & $3(6)$ \\
\hline
\end{tabular}

Numbers in parentheses are percentages (rounded). 


\section{RECANALISED SEGMENTS}

In addition to the stenoses already classified, 43 of the 54 cases had one or more arterial segments in which the lumen was multichannelled. Thirty three $(86.8 \%)$ of the 38 patients with old myocardial infarction had such segments as did $10(62.5 \%)$ of the 16 without old myocardial infarction $\left(\chi^{2}=2 \cdot 636, \mathrm{p}>0 \cdot 10\right)$.

\section{Discussion}

The present study confirms that in some segments of stenosed coronary artery in patients with stable angina there is the potential for the degree of obstruction to vary with vasomotor tone. When all the segments with stenosis of $>75 \%$ by cross sectional areas ( $>50 \%$ diameter) were considered, plaques in which an arc of normal media remained ( $\geqslant 16 \%$ of the perimeter of the residual lumen) were found in $15 \%$ of the 448 lesions. This figure is in broad agreement with two other studies that reported a frequency of $15 \%$ and $20 \% \cdot{ }^{613}$ It contrasts, however, with other studies in which $70 \%$ of high grade stenotic lesions had a vasospastic potential $^{3-5}$ and in which methods and definitions similar to those in the present study were used. The considerable variation in the distribution of plaque types from individual to individual, however, means that any figure based on pooled data gives no indication of the distribution in an individual. In some individuals all the plaques have a vasospastic potential, in others there are no such lesions. Twenty four of the 54 patients with stable angina in the present study had no lesions of this type. Patients with stable angina might be expected to have the lowest frequency of stenoses with vasospastic potential. Any study that includes patients with acute manifestations of ischaemic disease, in particular unstable angina, might have a far higher frequency of potentially vasospastic stenosis.

The potential for vasoconstriction within a stenotic segment depends on two morphological features. The first is the presence or absence of normal media. In arteries which have been distended to physiological pressures during fixation the plaque bulges outward and not inward (fig 10); the lumen is approximately round in cross section although the cross sectional area is considerably smaller than that of adjacent segments of normal artery. Behind the plaque the elastic lamina of the artery wall is often disrupted and the broken ends form coils at the margin of the plaque (fig 11); the media behind the plaque is thinned or even totally lost. In concentric plaques medial muscle may therefore be absent, whereas in eccentric plaques the arterial wall opposite the plaque has a normal elastic lamina and medial muscle of normal thickness and therefore a potential for vasoconstriction.

The second factor preventing vasoconstriction in concentric lesions is the splinting produced by intimal fibrosis and calcification (figs 3 and 4) that is effective even when medial muscle is retained. It is not known how thick the intima must be for the fibrosis to prevent alterations in lumen area with vasomotor tone; we have adopted the arbitrary definition of an intima that exceeds the media in

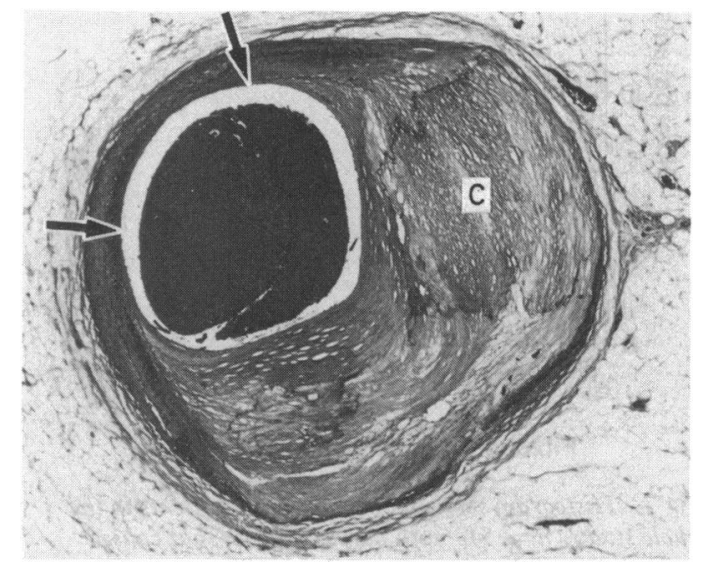

Fig 10 Photomicrograph showing stenosis caused by an eccentric fibrous plaque. The lumen is round because the bulk of the plaque has been extruded outward at the expense of the media. The plaque contains a plate of calcification (C). Opposite the plaque there is a large arc of normal vessel wall (arrows) over which the intima is normal. Elastical haematoxylin and eosin stain.

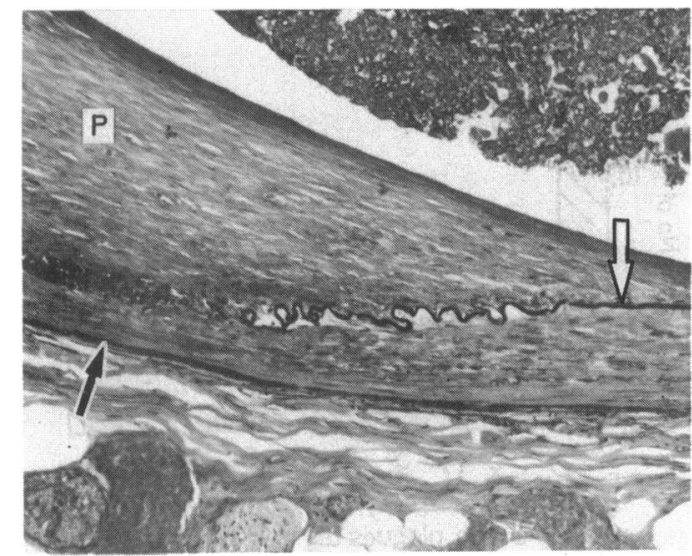

Fig 11 Photomicrograph showing the vessel wall at the margin of an eccentric plaque. Behind the plaque $(P)$ the media is thinned (black arrow). The internal elastic lamina is coiled and broken at the margin of the plaque. Within the arc of normal vessel wall the elastic lamina is straight and the media is of normal thickness (white arrow). Elastical haematoxylin and eosin stain. 
thickness. Clinical evidence, based on angiography, suggests that it is only eccentric lesions which vary in diameter with infusion of ergometrine and that the presence of an arc of normal vessel wall is the dominant determinant of the presence or absence of a vasospastic potential. ${ }^{1}$

There has been considerable discussion about the exact degree of pre-existing narrowing of the lumen in relation to the size of the arc of normal media around the residual lumen that can lead to vasospastic angina. ${ }^{237}$ To make our data comparable with previous studies we adopted the arbitary criterion that a segment of normal media occupying more than a $60^{\circ}$ arc of the vessel perimeter would give a potential for vasospasm. ${ }^{3}$ The theoretical basis for this figure is that in segments with $>75 \%$ stenosis by cross sectional area a shortening of $10 \%$ in the media will appreciably reduce flow. The calculation assumes that a $10 \%$ reduction in length is normally found when smooth muscle cells contract. Whereas this assumption may be true in physiological vasoconstriction, spasm ${ }^{14}$ could lead to far greater degrees of medial shortening and could create important obstruction at sites where the fixed stenotic element is $<75 \%$ by cross sectional area.

Where there is stenosis of $50-75 \%$ by cross sectional area $44 \%$ of lesions occur in arterial segments with a retained arc of medial muscle; most of these will be in series with segments of higher grade stenoses that are not capable of vasospasm.

A further and entirely unquantifiable variable is the physical properties of the plaque and the thickened intima adjacent to the plaque. Eccentric fibrous plaques may be less flexible than eccentric lipid rich plaques in which the intima contains a pool of lipid which, depending on its chemical composition, may or may not be in the liquid phase. ${ }^{15}$ Calcification could also splint the wall of the artery and prevent alteration in the cross sectional area of the lumen.

Postmortem studies cannot prove the existence of vasospasm in life; they can only demonstrate the presence or absence of plaques with morphological characteristics that make them capable of vasospasm. Selection of patients by different clinical criteria may specifically select patients with a preponderance of one type of plaque; thus it is feasible that a subgroup of patients with predominantly stable angina will have one or more lesions capable of variation in cross sectional area and responsible for episodes of ischaemia. There is clinical evidence to support this view; some patients with classic stable angina suffer a reduction in cross sectional area of stenotic segments on exercise while the normal vessel dilates. ${ }^{16}$ The pathological findings are consistent with the view that there is a clinical spectrum from patients with fixed stenosis who have angina precipitated only by a predictable level of exercise to patients who have angina precipitated at rest by variations in the cross sectional area of the vessel lumen. ${ }^{14}$

Differences in plaque morphology are relevant to the methods by which successful angioplasty may be achieved $^{45}$ and by which subsequent restenosis ${ }^{4}$ may occur. Generalisations about the relative frequencies of the types of plaque in a population with stable angina are of limited value because of the heterogeneity of the morphological types of plaques found in individuals. It is not possible to predict what types of plaques are present in an individual. Further, the characteristics of one plaque in an individual will not predict those of another plaque. Most patients with stable angina will have a mixture of all plaque types.

The presence or absence of plaques with a lipid pool may be relevant to individual prognosis. Plaques of this type have the greatest propensity to develop overlying thrombosis ${ }^{17}$ and thus acute infarction. Some patients with stable angina are at low risk because they do not have such plaques.

\section{References}

1 Lichtlen PR, Rafflenbeul W, Freudenberg H. Pathoanatomy and function of coronary obstructions leading to unstable angina pectoris; anatomical and angiographic studies. In: Hugenholz PG, Goldman BS, eds. Unstable angina. Stuttgart: Schattauer, 1985:81-93.

2 Brown BG, Bolson EL, Dodge HT. Dynamic mechanisms in human coronary stenosis. Circulation 1984; 70:917-22.

3 Freudenberg H, Lichtlen P. Das normale Wandsegment bei koronarstenosen eine postmortale Studie. $Z$ Kardiol 1981;70:863-9.

4 Saner HE, Gobel FL, Salomonowitz E, Erlien DA, Edwards JE. The disease free wall in coronary atherosclerosis: its relation to degree of obstruction. f Am Coll Cardiol 1985;6:1096-9.

5 Waller BF. Coronary luminal shape and the arc of disease free wall; morphologic observations and clinical relevance. F Am Coll Cardiol 1985;6:1100-1.

6 Quyyumi AA, Al-Rufaie HK, Olsen EGJ, Fox KM. Coronary anatomy in patients with various manifestations of three vessel coronary artery disease. Br Heart $\mathcal{F}$ 1985;54:362-6.

7 Brown BG. Coronary vasospasm: observations linking the clinical spectrum of ischemic heart disease to the dynamic pathology of coronary atherosclerosis. Arch Intern Med 1981;141:716-22.

8 Shea MJ, Deanfield JE, Wilson R, Delandsheere C, Jones T, Selwyn AP. Transient ischemia in angina pectoris: frequent silent events with everyday activities. Am $\mathcal{F}$ Cardiol 1985;56:34E-8E.

9 Block PC. Mechanism of transluminal angioplasty. Am f Cardiol 1984;53:69C-71C. 
10 Davies MJ, Thomas AC. Thrombosis and acute coronary artery lesions in sudden cardiac ischaemic death. $N$ Engl f Med 1984;310:1137-40.

11 Thomas AC, Davies MJ. Postmortem investigation and quantification of coronary artery disease. Histopathology 1985;9:959-76.

12 Brandt PWT, Partridge JB, Wattie WJ. Coronary arteriography: method of presentation of arteriogram report and a scoring system. Clin Radiol 1977; 28:361-5.

13 Hort W, Moosdorf R, Kalbfleisch H, Kohler F, Milzner-Schwarz U, Frenzel H. Postmortale Untersuchungen uber Lokalisation und Form der stärksten Stenosen in den Koronararterien und ihre Beziehung zu den Risikofaktoren. $Z$ Kardiol
1977;66:333-40.

14 Maseri A, Chierchia S, Kaski JC. Mixed angina pectoris. Am $\mathcal{f}$ Cardiol 1985;56:30E-3E.

15 Lundberg B. Chemical composition and physical state of lipid deposits in atherosclerosis. Atherosclerosis 1985;56:93-110.

16 Gage JE, Hess OM, Murakami T, Ritter M, Grimm J, Krayenbuehl HP. Vasoconstriction of stenotic coronary arteries during dynamic exercise in patients with classic angina pectoris: reversibility by nitroglycerin. Circulation 1986;73:865-76.

17 Davies MJ, Thomas AC. Plaque fissuring-the cause of acute myocardial infarction, sudden ischaemic death and crescendo angina. Br Heart $\mathcal{F}$ 1985;53: 363-73. 